\title{
Work-Related Injuries Among Certified Nursing Assistants Working in US Nursing Homes
}

Galina Khatutsky, Joshua M. Wiener, Wayne L. Anderson, and Frank W. Porell

April 2012 


\section{About the Authors}

Galina Khatutsky, MS, is a health services researcher in the Aging, Disability, and Long-Term Care

Program at RTI International.

Joshua M. Wiener, PhD, is a Distinguished Fellow and program director for Aging, Disability and Long-Term Care at RTI International.

Wayne L. Anderson, PhD, is a senior research economist in RTI's Aging, Disability and Long-Term Care Program.

Frank W. Porell, PhD, is Professor of Gerontology, McCormack Graduate School, the University of Massachusetts, Boston.
RTI Press publication RR-0017-1204

This PDF document was made available from www.rti.org as a public service of RTI International. More information about RTI Press can be found at http://www.rti.org/rtipress.

RTI International is an independent, nonprofit research organization dedicated to improving the human condition by turning knowledge into practice. The RTI Press mission is to disseminate information about RTI research, analytic tools, and technical expertise to a national and international audience. RTI Press publications are peer-reviewed by at least two independent substantive experts and one or more Press editors.

\section{Suggested Citation}

Khatutsky, G., Wiener, J. M., Anderson, W. L., \& Porell, F.W. (2012). Work-related injuries among certified nursing assistants working in US nursing homes. RTI Press publication No. RR-0017-1204. Research Triangle Park, NC: RTI Press. Retrieved from www.rti.org/rtipress.
This publication is part of the RTI Press Research Report series.

RTI International

3040 Cornwallis Road

PO Box 12194

Research Triangle Park, NC

27709-2194 USA

919.541 .6000

rtipress@rti.org

www.rti.org
C2012 Research Triangle Institute.

RTI International is a trade name of Research Triangle Institute.

All rights reserved. This report is protected by copyright. Credit must be provided to the author and source of the document when the content is quoted. Neither the document nor partial or entire reproductions may be sold without prior written permission from the publisher.

doi:10.3768/rtipress.2012.rr.0017.1204

www.rti.org/rtipress 


\title{
Work-Related Injuries Among \\ Certified Nursing Assistants \\ Working in US Nursing Homes
}

\begin{tabular}{lr} 
Contents & \\
Introduction & 2 \\
Methods & 2 \\
Findings & 4 \\
\multicolumn{1}{c}{ Descriptive Analysis } & 4 \\
$\quad$ Multivariate Analysis & 5 \\
Discussion & 10 \\
References & 12 \\
Acknowledgments inside back cover
\end{tabular}

Galina Khatutsky, Joshua M. Wiener, Wayne L. Anderson, and Frank W. Porell

Acknowledgments inside back cover

\begin{abstract}
Certified nursing assistants (CNAs) working in nursing homes are at significant risk for work-related injuries, but little is known about the frequency and types of such injuries and how assistive equipment such as patient lifts affect injury rates. This study uses 2004 data from the National Nursing Assistant Survey and the National Nursing Home Survey to analyze the prevalence, nature, and predictors of these injuries among CNAs working in US nursing homes. The study found that 60.2 percent of all CNAs nationally reported a work-related injury in the year prior to the survey; among injured CNAs, 65.8 percent reported being injured more than once in the past year, 16 percent required a transfer to light duty work, and 24 percent were unable to work because of their injury. The reported injuries varied in nature and included scratches, open wounds, back injuries, black eyes and other bruising, human bites, and strained or pulled muscles. In examining predictors of injury, the study found that although assistive equipment was readily available and often used, it was not associated with lower rates of workforce injuries. New workers, workers who change jobs more frequently, those reporting poor job preparation, workers who received lower wages, workers who felt that they had inadequate time to provide personal care, and those working mandatory overtime were more likely to have a workplace injury. CNAs who worked in facilities where they felt respected and rewarded for their work and where the organization valued their work were less likely to report an injury.
\end{abstract}




\section{Introduction}

Direct care workers represent the backbone of the long-term care workforce (Stone \& Wiener, 2001; Wiener, 2006). Over 600,000 certified nursing assistants (CNAs) provide the majority of daily care to more than 1.4 million nursing home residents (Bureau of Labor Statistics [BLS], 2007). CNAs help residents with the activities of daily living such as bathing, eating, dressing, toileting, and transferring in and out of a bed or chair.

There is a growing shortage of qualified long-term care workers who are able to meet the needs of older adults (Stone \& Harahan, 2010). In part, this is due to the high physical and emotional demands and the significant potential for on-the-job injury (Institute of Medicine, 2008). In 2010, the Bureau of Labor Statistics (BLS) reported the incidence rate of 8.3 nonfatal injuries for every 100 workers in nursing and residential care facilities, of which 4.9 injuries per 100 workers resulted in days away from work, job transfer, or restriction in activities (BLS, 2011).The most recent BLS data show that workers in nursing and residential care facilities now have the highest injury rates of any occupational setting, moving up from third place, after construction workers and truck drivers, their rank in 2006 (BLS 2006, 2011). Large workloads, an inadequate supply of assistive equipment, and lack of training are commonly cited factors contributing to high injury rates among CNAs (Hoskins, 2006).

Although injuries commonly occur among CNAs working in nursing homes, little is known about the nature and causes of various types of injuries. In addition, it is not clear whether assistive equipment affects injury rates and whether workers receive adequate training in using such equipment. Aside from the impact on workers' health, work-related injuries have a direct effect on lost work time and may affect CNAs' job tenure and satisfaction.

The main purpose of our study was to analyze the determinants of work-related injuries among CNAs who work in nursing homes.
Our goals were to answer the following research questions:

- What is the prevalence and impact of workforce injuries in nursing homes?

- What assistive equipment is available in nursing facilities, and how is it used?

- What is the extent and quality of training that CNAs receive in injury prevention and use of assistive equipment?

- What personal, facility, and workload characteristics increase the likelihood of being injured?

\section{Methods}

Our study linked 2004 data from the National Nursing Home Survey (NNHS) and the National Nursing Assistant Survey (NNAS). These surveys are the most recent data available that combine detailed responses from CNAs with detailed information about the nursing homes in which they work. We used the NNHS, which serves as the sampling frame for the NNAS, to obtain facilitylevel data about the organizations for which the CNAs worked. The NNHS is a national probability survey of nursing facilities conducted periodically by the National Center for Health Statistics (NCHS) (Jones, Dwyer, Bercovitz, \& Strahan, 2009). The NNHS sample included 1,500 nursing facilities selected using systematic sampling with probability proportional to their bed size. Additionally, we used the average activities of daily living (ADL) score for facility residents derived from the Online Survey, Certification, and Reporting (OSCAR) system.

The NNAS, sponsored by the Department of Health and Human Services' Office of the Assistant Secretary for Planning and Evaluation and conducted in partnership with the NCHS, is the first nationally representative survey of CNAs working in nursing homes (Squillace, Remsburg, Bercovitz, Rosenoff, \& Branden, 2007). The survey provides information on CNAs' personal characteristics, work experience, reasons for entering the field, job characteristics and rewards, working conditions, job satisfaction, why they leave the field, and injury experience. The NNAS sample used a two-stage probability design. In the first stage, a randomly selected subsample of 790 of 
the 1,500 nursing homes in the NNHS sample was selected to participate in the NNAS. Of these, 208 facilities were deleted from the sample for ineligibility or nonparticipation in the NNHS or NNAS. The remaining 582 facilities provided a list of CNAs for sampling, yielding a facility response rate of 76 percent.

In the second stage, a sample of CNAs was selected randomly from each of the 582 facilities. In total, 4,542 CNAs were selected to participate in the NNAS. Of those selected, 4,274 were eligible and 3,017 completed an interview, yielding a response rate of 71 percent among those eligible and a combined NNHS-NNAS response rate of 53 percent (Squillace et al., 2007).

Data were collected through telephone interviews between September 2004 and January 2005. The sample of 3,017 respondents, when weighted, represents 680,800 CNAs employed during the NNAS. The study sample of 2,886 CNAs represents all respondents for whom we have valid survey data on workplace injuries.

In the analysis, a measure for a work-related injury was created from NNAS survey items on work-related injuries occurring at the sampled facility over the past year. The injuries reported by survey respondents included back injury, strained or pulled muscles, human bites, scratches, open wounds, cuts, black eyes or other bruising, or any other injury. The NNAS does not include questions on whether the injuries were reported to the facility management, but it excludes minor injuries from the overall injury count.

The study includes descriptive and multivariate analyses. Our major hypothesis is that availability and use of lifting equipment reduces the likelihood of CNA injures. Although not entirely consistent in its findings, past research suggests that assistive equipment and employee training to use it reduce work-related injuries in health care. For example, one study showed that assistive equipment, such as patient lifts, helps reduce the incidence of back injuries among nurses (Silverwood \& Haddock, 2006). In contrast, a study on injuries in Ohio nursing homes found that the absolute number of safety devices in facilities had little or no relationship to the rate of employee injury (Stanev, Bailer, \& Li, 2008).

Other factors are also potentially important, including training, workload, mandatory overtime, and organizational culture. Training on injury prevention, as part of a larger intervention, led to significant reductions in workers' compensation costs and lost work days (Collins, Wolf, Bell, \& Evanoff, 2004). Trinkoff, Johantgen, Muntaner, and Le demonstrated that low staffing ratios were associated with higher prevalence of work-related injuries (Trinkoff et al., 2005). Similarly, job overload factors were found to be a significant predictor of work-related injuries (Eriksen, Bruusgaard, \& Knardahl, 2003; Horwitz \& McCall, 2004).

In the multivariate model we include variables indicating the number of hours worked per week, whether mandatory overtime work is required, and whether CNAs reported not having enough time to provide personal care and to perform other duties. We hypothesized that a positive, supportive, and innovative organizational culture in the facility would lead to a lower risk of injury. Existing research also influenced selection of facility-level variables. We included an indicator for a special dementia unit because there is evidence that the presence of these units reduces work-related injuries (Morgan, Stewart, D’Arcy, Forbes, \& Lawson. 2005). Castle, Engberg, Mendeloff, and Burns (2009) found chain status to be predictive of higher rates of staff injury in a facility.

We also hypothesized that certain work-related CNA characteristics such as being a new worker in the facility, being new to the occupation, and having changed jobs more than twice in the last 5 years increase the probability of injury. Workers who feel their initial training in the facility poorly prepared them for the job may also have an increased likelihood of getting injured. Prior descriptive analyses found that CNA injury rates vary by demographic characteristics such as age, non-white race, and immigrant status and by levels of education (Khatutsky, Anderson, Wiener, \& Akhmerova, 2007); other CNA demographic characteristics were included in the model as control variables. 


\section{Findings}

\section{Descriptive Analysis}

Table 1 presents the summary descriptive statistics on CNA injuries. Nationally, 60.2 percent of CNAs reported having a work-related injury in the year before the survey; 57.9 percent of CNAs reported a non-minor work-related injury. Among those with non-minor injuries, CNAs experienced an average of 4.5 injuries per person per year, although the median number of injuries was less than half the mean (the number of reported injuries ranged from 1 to 50, with a median of 2). A large number of CNAs whose

\section{Table 1. Work-related injuries among CNAs in nursing homes}

\begin{tabular}{|c|c|}
\hline \multirow[t]{2}{*}{$\mathbf{N}$ (unweighted) } & 2,886 \\
\hline & Percent \\
\hline $\begin{array}{l}\text { CNAs reporting any injury in the past year } \\
(n=1,738)\end{array}$ & 60.2 \\
\hline \multicolumn{2}{|l|}{$\begin{array}{l}\text { Types of injuries received in past year among } \\
\text { injured CNAs }(n=1,738)\end{array}$} \\
\hline Scratches, open wounds, and cuts & 44.6 \\
\hline Back injuries & 17.5 \\
\hline Black eyes, other bruising & 16.2 \\
\hline $\begin{array}{l}\text { Strained or pulled muscles (other than back } \\
\text { injuries) }\end{array}$ & 15.7 \\
\hline Human bites & 11.5 \\
\hline Other injuriesa & 7.2 \\
\hline \multicolumn{2}{|l|}{ Job restrictions among injured CNAs $(n=1,738)$} \\
\hline Restricted duty offered because of injury & 15.9 \\
\hline Unable to work & 24.2 \\
\hline $\begin{array}{l}\text { Total days on restricted duty because of injuries } \\
\text { (mean) }\end{array}$ & 20.1 \\
\hline $\begin{array}{l}\text { Total days unable to work because of injuries } \\
\text { (mean) }\end{array}$ & 3.0 \\
\hline CNAs reporting non-minor injuries ${ }^{b}(N=1,670)$ & 57.9 \\
\hline CNAs injured more than once in the past year & 65.8 \\
\hline CNAs injured 10 times or more in the past year & 11.7 \\
\hline Average number of non-minor injuries (mean) & 4.5 \\
\hline \multicolumn{2}{|c|}{$\begin{array}{l}\text { a Other injuries include hits, kicks, being punched or grabbed, having } \\
\text { dislocations or joint injuries, and being run over, bumped, or crashed into. }\end{array}$} \\
\hline \multicolumn{2}{|c|}{$\begin{array}{l}\text { b Some of the injuries reported by CNAs were considered minor and were } \\
\text { excluded from the count of injuries. }\end{array}$} \\
\hline \multicolumn{2}{|c|}{$\begin{array}{l}\text { Notes: All means and proportions are weighted estimates. Unweighted Ns are } \\
\text { provided for reference. }\end{array}$} \\
\hline \multicolumn{2}{|c|}{$\begin{array}{l}\text { Source: RTI analysis of the } 2004 \text { National Nursing Home Survey (NNHS) and } \\
2004 \text { National Nursing Assistant Survey (NNAS). }\end{array}$} \\
\hline
\end{tabular}

injuries were not minor (65.8 percent) reported multiple injuries per year; 11.7 percent reported 10 injuries or more. (In our sample, 40 CNAs reported 50 or more injuries. Because this was not a trivial number of respondents, we did not exclude these reports.)

The injuries were of various levels of severity. Almost 16 percent of injured CNAs reported a transfer to restricted or light duty at work for an average of about 20 days. Almost a quarter of injured CNAs reported being unable to work because of their injury, missing about 3 days of work on average.

CNA injuries included scratches, open wounds, and cuts (44.6 percent of injured CNAs reported these); back injuries (17.5 percent); black eyes and other bruising (16.2 percent); strained or pulled muscles, other than back strain (15.7 percent); and human bites ( 11.5 percent). It appears that a substantial portion of the injuries are from aggressive residents (e.g., black eyes and human bites).

Seven percent of those injured reported other types of injuries, including hits, kicks, being punched or grabbed, having dislocations or joint injuries, and being run over, bumped, or crashed into. Of all types of injuries, back injuries, other strains and pulled muscles, and open wounds, cuts, and scratches result in most job restrictions (data not shown). CNAs also reported that injuries happened during lifting, bathing, and handling residents; from bumping and hitting equipment; and from tripping, slipping, and falling.

Lifting residents is one of the main causes of injuries in nursing homes. To reduce back strain and musculoskeletal injuries among direct care stuff, various types of mechanical assistive equipment for lifting patients are used in nursing facilities. Table 2 presents descriptive statistics on the availability and use of assistive equipment as well as statistics on training on injury prevention and equipment use.

Overall, assistive equipment is widely available. Most CNAs (87.6 percent) reported that lifting devices are always available, and 10.3 percent reported that they are sometimes available. Only a few CNAs reported lack of access to assistive equipment. Fully 60.6 


\section{Table 2. Assistive equipment use among CNAs in nursing homes}

\begin{tabular}{|c|c|}
\hline \multirow[t]{2}{*}{ N (unweighted) } & 2,886 \\
\hline & Percent \\
\hline \multicolumn{2}{|l|}{ CNA uses lifting devices } \\
\hline Always & 60.6 \\
\hline Sometimes & 34.1 \\
\hline Never & 5.3 \\
\hline \multicolumn{2}{|l|}{ Lifting devices available when needed } \\
\hline Always & 87.6 \\
\hline Sometimes & 10.3 \\
\hline Almost never or never & 2.1 \\
\hline \multicolumn{2}{|l|}{ Training } \\
\hline Received training to use lifting devices & 99.1 \\
\hline $\begin{array}{l}\text { Facility provides training to reduce workplace } \\
\text { injuries }\end{array}$ & 92.2 \\
\hline \multicolumn{2}{|l|}{ Equipment needs } \\
\hline $\begin{array}{l}\text { Equipment other than lifting device needed to } \\
\text { make job safer }\end{array}$ & 15.9 \\
\hline \multicolumn{2}{|l|}{ Type of equipment needed $(n=459)$} \\
\hline Bathing aidsa & 13.9 \\
\hline Electric beds & 6.9 \\
\hline Belts-walking/gait belts & 11.0 \\
\hline Belts-back & 6.9 \\
\hline Other & 86.1 \\
\hline \multicolumn{2}{|c|}{$\begin{array}{l}\text { Bathing aides include shower chairs, adapted shower stalls, and bathing } \\
\text { systems. }\end{array}$} \\
\hline \multicolumn{2}{|c|}{$\begin{array}{l}\text { Notes: All proportions are weighted estimates. Unweighted Ns are provided } \\
\text { for reference. }\end{array}$} \\
\hline $\begin{array}{l}\text { Source: RTI analysis of the } 2004 \text { National Nursing Home Sur } \\
2004 \text { National Nursing Assistant Survey (NNAS). }\end{array}$ & -IS) and \\
\hline
\end{tabular}

percent of CNAs reported that they always use lifting devices, about 34.1 percent say that they sometimes use them, and only a few say that they never use them. However, 15.9 percent of CNAs also reported that they need additional equipment such as bathing aids, ${ }^{1}$ electric beds, and walking/gait ${ }^{2}$ and back belts ${ }^{3}$ to make their jobs safer. Other equipment mentioned

1 Bathing aides include shower chairs, adapted shower stalls, and bathing systems.

2 Walking/gait belts are devices used to transfer people from one position to another, such as from a standing position to a wheelchair. The gait belt is worn around the nursing home resident's waist. The purpose of the walking/gait belt is to put less strain on the back of the caregiver and to provide support for residents.

3 Worn by CNAs, back belts are lightweight belts worn around the lower back to provide support to the lumbar region to aid in lifting. included specific kinds of lifts and lifting equipment and blood pressure and vital signs monitors.

Interestingly, it appears that equipment use is not necessarily driven by its availability; there are other reasons CNAs are not using the equipment. Of the 1,026 CNAs who reported using lifting devices sometimes, only 35 also reported that the devices are almost never or never available (716 of these CNAs reported that these devices are always available, and 275 reported that they are sometimes available). Similarly, of 164 CNAs who reported never using equipment, only 35 also said that devices are almost never or never available and the rest reported good access to equipment.

Federal regulations mandate that CNAs receive at least 75 hours of training, with a 16-hour clinical component (Wright, 2006). It appears that almost all facilities nationwide are providing some training to CNAs to prevent injuries-99.1 percent of CNAs received training on lifting devices, and 92.2 percent reported receiving general training to reduce workplace injuries at a nursing facility. On the other hand, other studies using NNAS data show that more than a third of all respondents stated that they feel "not at all prepared" or only "somewhat prepared" overall for their jobs in a nursing facility (Khatutsky et al., 2007).

\section{Multivariate Analysis}

We estimated a multivariate model to examine what factors increase or decrease the risk of CNAs' being injured on the job. The predictors were grouped into the following domains:

CNA personal characteristics: CNA demographics included age, racial and immigrant status, and educational attainment. Other personal characteristics included receipt of public benefits as a proxy for income and conflicting family obligations such as family caregiving responsibilities.

Lift equipment use: Variables included CNA-reported lifting device use and availability in the facility and CNAs' perceptions on how their initial training prepared them to avoid work-related injuries. 
CNA job characteristics: Characteristics of the CNAs' work environment included extrinsic rewards (e.g., wages and benefits) and tenure in that position and in the field of nursing assistance. The model also includes variables indicating job overload, such as number of hours worked per week, whether CNAs worked mandatory overtime, and whether they reported inadequate time for helping residents with personal care and other duties.

Perception of organizational culture: Organizational culture as perceived by CNAs was measured by including CNAs' perspectives on being supported by coworkers, feeling respected and valued, and being considered as a part of the health care team; the degree of autonomy and challenge in their work; and whether CNAs reported having permanent assignments of particular residents to care for.

Facility characteristics: These variables included bed count, ownership and chain status, presence of special care dementia units, CNA staffing ratio, CNA union status, average resident frailty score, and number of lifting devices per 10 residents (a facility-level measure that is different from CNAs' reports on use and availability of lifting equipment).

Table 3 describes the study sample and presents weighted means, standard errors of the mean, and 95 percent confidence levels for the mean for all variables in the model. The regression analysis sample includes 2,461 observations for which all variables have non-missing data. Overall, CNAs were fairly equally distributed among three age groups: 30 percent were under the age of 30, 36 percent were between the ages of 30 and 45, and 34 percent were aged 46 and over. Only 8 percent of CNAs were male; slightly less than half reported being non-white. CNAs had low educational attainment (three-quarters of CNAs had a high school education or less) and low incomes (about a third reported receiving some kind of a public assistance, such as Medicaid, Supplemental Nutrition Assistance Program, or rental assistance). On average, they reported receiving $\$ 10.40$ per hour in wages and working about 37 hours a week; 22 percent reported working mandatory overtime. About 40 percent of all CNAs were new workers (i.e., CNAs who worked in the facility for less than a year and were also new to the CNA occupation). CNAs change their jobs often: about 73 percent reported having two or more jobs in the past 5 years. More than 40 percent of all CNAs reported not having enough time for delivering personal ADL care to residents. About a third of the respondents reported having been poorly or somewhat poorly prepared for their job in a nursing facility. Most CNAs had a positive perception of the organizational culture of the facilities in which they work.

Table 3. Predicting the probability of work-related injuries: sample means

\begin{tabular}{|c|c|c|c|c|}
\hline Variable & $\begin{array}{l}\text { Weighted } \\
\text { Mean }\end{array}$ & $\begin{array}{l}\text { Standard } \\
\text { Error of the } \\
\text { Mean }\end{array}$ & \multicolumn{2}{|c|}{$95 \%$ Cl for Mean } \\
\hline \multicolumn{5}{|l|}{$\mathrm{N}=2,461$} \\
\hline All injuries & 0.59 & 0.014 & 0.57 & 0.62 \\
\hline \multicolumn{5}{|l|}{ Lift equipment use } \\
\hline Lift equipment use (always) & 0.60 & 0.016 & 0.57 & 0.63 \\
\hline Lifting device (always available) & 0.87 & 0.010 & 0.85 & 0.89 \\
\hline Initial training preparation to avoid injuries (excellent/good) & 0.90 & 0.008 & 0.89 & 0.92 \\
\hline \multicolumn{5}{|l|}{ CNA personal characteristics } \\
\hline Age under 30 & 0.30 & 0.013 & 0.28 & 0.33 \\
\hline Age over 45 & 0.34 & 0.014 & 0.31 & 0.36 \\
\hline Male gender & 0.08 & 0.007 & 0.06 & 0.09 \\
\hline Non-white race & 0.46 & 0.020 & 0.42 & 0.50 \\
\hline
\end{tabular}


Table 3. Predicting the probability of work-related injuries: sample means (continued)

\begin{tabular}{|c|c|c|c|c|}
\hline Variable & $\begin{array}{l}\text { Weighted } \\
\text { Mean }\end{array}$ & $\begin{array}{l}\text { Standard } \\
\text { Error of the } \\
\text { Mean }\end{array}$ & \multicolumn{2}{|c|}{$95 \% \mathrm{Cl}$ for Mean } \\
\hline \multicolumn{5}{|l|}{ CNA personal characteristics (continued) } \\
\hline Less than high school/high school education & 0.75 & 0.013 & 0.72 & 0.77 \\
\hline Married & 0.51 & 0.014 & 0.49 & 0.54 \\
\hline Immigrant & 0.19 & 0.018 & 0.16 & 0.23 \\
\hline Any public benefit receipt & 0.31 & 0.013 & 0.29 & 0.34 \\
\hline CNA caring for a child or family/friend with a disability & 0.39 & 0.014 & 0.36 & 0.42 \\
\hline \multicolumn{5}{|l|}{ CNA job characteristics } \\
\hline Wages & $\$ 10.4$ & $\$ 0.1$ & $\$ 10.2$ & $\$ 10.6$ \\
\hline New worker & 0.39 & 0.014 & 0.36 & 0.42 \\
\hline Tenure in the field 12 months or more & 0.89 & 0.008 & 0.87 & 0.90 \\
\hline Two or more jobs in the past 5 years & 0.73 & 0.013 & 0.71 & 0.76 \\
\hline Required to work mandatory overtime & 0.22 & 0.017 & 0.18 & 0.25 \\
\hline Not enough time for personal care & 0.43 & 0.015 & 0.40 & 0.46 \\
\hline Not enough time for other tasks & 0.44 & 0.015 & 0.41 & 0.47 \\
\hline Number of hours work per week & 36.77 & 0.232 & 36.32 & 37.23 \\
\hline Job preparation is poor & 0.34 & 0.013 & 0.32 & 0.37 \\
\hline \multicolumn{5}{|l|}{ Perception of organizational culture } \\
\hline Coworkers a reason to stay on the job & 0.77 & 0.012 & 0.75 & 0.80 \\
\hline CNAs feel respected/rewarded for their work & 0.69 & 0.013 & 0.67 & 0.72 \\
\hline CNA has opportunity to work in teams & 0.83 & 0.012 & 0.81 & 0.86 \\
\hline CNAs can decide how to do their work & 0.90 & 0.009 & 0.88 & 0.92 \\
\hline Organization values CNA work & 0.92 & 0.007 & 0.91 & 0.94 \\
\hline CNA is involved in challenging work & 0.92 & 0.007 & 0.91 & 0.94 \\
\hline Supervisor is the reason to stay on the job & 0.62 & 0.013 & 0.60 & 0.65 \\
\hline Permanent resident assignment & 0.48 & 0.017 & 0.44 & 0.51 \\
\hline \multicolumn{5}{|l|}{ Facility characteristics } \\
\hline Facility size (large) & 0.67 & 0.013 & 0.65 & 0.70 \\
\hline Facility ownership (profit) & 0.58 & 0.027 & 0.53 & 0.64 \\
\hline Dementia care unit & 0.39 & 0.026 & 0.34 & 0.44 \\
\hline Chain status & 0.54 & 0.026 & 0.49 & 0.59 \\
\hline CNA staffing ratio (hours per patient per day) & 2.36 & 0.086 & 2.19 & 2.52 \\
\hline Average ADL score for facility residents (0-6) & 5.86 & 0.029 & 5.80 & 5.92 \\
\hline No. lifting devices per 10 beds & 5.82 & 0.241 & 5.35 & 6.30 \\
\hline Number of nursing staff injured at facility (sick leave or light duty) & 1.33 & 0.176 & 0.98 & 1.67 \\
\hline Over $40 \%$ of residents on Medicaid & 0.88 & 0.017 & 0.85 & 0.91 \\
\hline CNAs belong to union & 0.21 & 0.023 & 0.17 & 0.26 \\
\hline
\end{tabular}

Note: All analyses are weighted estimates and adjusted for complex survey design.

Source: RTI analysis of the 2004 National Nursing Home Survey (NNHS) and 2004 National Nursing Assistant Survey (NNAS). 
Table 4 presents the results of the multivariate analysis. Among policy variables, organizational culture and CNA job characteristics were the most important predictors of work-related injuries, whereas measures related to the use and availability of assistive equipment were not statistically significant. This result is surprising given the emphasis that policymakers and providers place on using this equipment as a strategy to reduce workplace injuries. For CNAs working in nursing facilities, policyrelevant factors decreasing the odds of being injured in the past year included receiving higher wages, feeling respected and rewarded for their work, and working for a facility they perceived as valuing CNA work.
Several factors increased the odds of being injured on the job. Respondents who were new to the CNA occupation and had worked in the facility for less than 1 year had a 30 percent higher likelihood of being injured. CNAs reporting two or more jobs in the past 5 years increased their odds of injury by about 50 percent. Being required to work overtime increased the odds of being injured by nearly 80 percent. CNAs reporting not having enough time for providing personal care (helping nursing home residents with their ADLs), and those who rated their initial training for working in nursing homes as fair/poor had a greater than 30 percent increased likelihood of being injured.

Table 4. Predictors of work-related injuries among CNAs working in nursing homes: multivariate analysis

$\mathrm{N}=\mathbf{2 , 4 6 1}$

\begin{tabular}{|c|c|c|c|c|}
\hline Lift equipment use & & & & \\
\hline Always use lifting device & 1.17 & 0.88 & 1.55 & \\
\hline Lifting device always available & 1.10 & 0.73 & 1.65 & \\
\hline Initial training preparation to avoid injuries (excellent/good) & 0.70 & 0.47 & 1.03 & \\
\hline CNA personal characteristics & & & & \\
\hline Age under 30 & 1.62 & 1.20 & 2.21 & $* *$ \\
\hline Age over 45 & 1.41 & 1.07 & 1.86 & * \\
\hline Male gender & 0.58 & 0.40 & 0.84 & $* *$ \\
\hline Non-white race & 0.53 & 0.40 & 0.70 & *** \\
\hline Less than high school/high school education & 0.71 & 0.54 & 0.94 & * \\
\hline Married & 1.07 & 0.82 & 1.40 & \\
\hline Immigrant & 1.09 & 0.75 & 1.58 & \\
\hline Any public benefit receipt & 1.17 & 0.89 & 1.54 & \\
\hline CNA caring for a child or family/friend with a disability & 0.85 & 0.66 & 1.09 & \\
\hline CNA job characteristics & & & & \\
\hline Wages $\$$ per hour & 0.91 & 0.85 & 0.97 & ** \\
\hline New worker & 1.31 & 1.02 & 1.69 & * \\
\hline Tenure in the field 12 months or more & 1.07 & 0.75 & 1.51 & \\
\hline Two or more jobs in the past 5 years & 1.47 & 1.05 & 2.05 & * \\
\hline Required to work mandatory overtime & 1.79 & 1.29 & 2.49 & *** \\
\hline Not enough time for personal care & 1.34 & 1.02 & 1.76 & * \\
\hline Not enough time for other tasks & 1.22 & 0.92 & 1.61 & \\
\hline Number of hours work per week & 1.00 & 0.99 & 1.02 & \\
\hline Job preparation is poor & 1.35 & 1.05 & 1.73 & * \\
\hline
\end{tabular}




\begin{tabular}{|c|c|c|c|c|}
\hline Variable & Odds Ratio & \multicolumn{2}{|c|}{ 95\% Confidence Limits } & Sign \\
\hline \multicolumn{5}{|l|}{ Perception of organizational culture } \\
\hline Coworkers a reason to stay on the job & 0.92 & 0.69 & 1.23 & \\
\hline CNAs feel respected/rewarded for their work & 0.66 & 0.49 & 0.88 & $* *$ \\
\hline CNAs has opportunity to work in teams & 1.05 & 0.74 & 1.49 & \\
\hline CNAs can decide how to do their work & 0.97 & 0.62 & 1.53 & \\
\hline Organization values CNA work & 0.51 & 0.28 & 0.94 & * \\
\hline CNA is involved in challenging work & 0.64 & 0.41 & 1.01 & \\
\hline Supervisor is the reason to stay on the job & 1.02 & 0.78 & 1.32 & \\
\hline Permanent resident assignment & 0.96 & 0.76 & 1.20 & \\
\hline \multicolumn{5}{|l|}{ Facility characteristics } \\
\hline Facility bed size (large) & 0.93 & 0.71 & 1.21 & \\
\hline Facility ownership (for profit) & 1.07 & 0.80 & 1.44 & \\
\hline Dementia care unit & 1.28 & 0.95 & 1.73 & \\
\hline Chain status & 1.06 & 0.82 & 1.39 & \\
\hline CNA staffing ratio (hours per patient per day) & 0.97 & 0.93 & 1.02 & \\
\hline Average ADL score for facility residents (0-6) & 0.91 & 0.73 & 1.15 & \\
\hline Number of lifting devices per 10 beds & 1.00 & 0.98 & 1.03 & \\
\hline Number of nursing staff injured at facility (sick leave/light duty) & 1.02 & 0.96 & 1.07 & \\
\hline Over $40 \%$ of residents on Medicaid & 0.95 & 0.60 & 1.52 & \\
\hline CNAs belong to union & 0.80 & 0.54 & 1.17 & \\
\hline \multicolumn{5}{|l|}{ Model } \\
\hline Pseudo-R2 & 0.102 & & & \\
\hline c statistic & 0.680 & & & \\
\hline
\end{tabular}

${ }^{*} p<.05,{ }^{* *} p<.01$, and ${ }^{* * *} p<.001$.

Note: All analyses are weighted estimates and adjusted for complex survey design.

Source: RTI analysis of the 2004 National Nursing Home Survey (NNHS) and 2004 National Nursing Assistant Survey (NNAS).

In addition to policy-relevant factors, several CNA personal characteristics were significant predictors and were associated with increased odds of injury; these factors included being younger than 30 or more than 45 years old, being female, being white, and being better educated. Age appears to be an important predictor of injuries. The findings indicate that CNAs on both ends of the age spectrum-those younger and older-tend to get injured more often than those aged 31 to 45 . Males and minorities received fewer injuries of all types. CNAs with lower levels of education had a lower risk of being injured.

Contrary to our expectations, several factors that we hypothesized to affect the probability of being injured on the job were not statistically significant predictors. Along with the previously mentioned nonsignificant findings on the availability and use of lifting devices, resident acuity levels, facility chain status and ownership type, and the presence of special dementia units in a facility were not significant predictors of injury. Moreover, CNA staffing ratio was not a significant predictor of injury. We also did not find significant effects on injuries among other organizational culture variables such as CNA reports of consistent staff assignment to the same residents, working in teams, and CNA participation in decisionmaking.

As part of a larger study on worker injuries in nursing homes, we also examined injuries by type, seeking to understand whether different factors predict 
musculoskeletal injuries compared to predictors of all other types of injuries such as scratches, open wounds, black eyes, bruising, bites, and others that are not classified as musculoskeletal trauma and may be related to accidents and resident aggression (data not shown). Overall, 25 percent of CNAs report suffering musculoskeletal injuries and 34 percent report suffering all other types of injuries. We found that no other specific factors are predictive of musculoskeletal injuries. As for all types of injuries, CNAs who were new to the profession and to the facility, as well as those working mandatory overtime and those who rated their initial training as poor preparation for their work in the facility, were at higher odds of developing musculoskeletal injuries. Assistive equipment availability and use were not significant predictors of either musculoskeletal or other types of injuries once CNA personal and work characteristics were controlled for.

\section{Discussion}

This study found that work-related injuries are extremely prevalent among CNAs working in US nursing homes and that most CNAs report multiple injuries. Injuries vary in nature ranging from back injuries to human bites, and are the result of a variety of causes, including lifting, bathing, and handling residents; resident aggression; and accidents involving facility equipment. In considering the prevalence rates found in this study, it is important to remember that the injuries are self-reported and are not limited to serious injuries that are reported to facility management and result in workers' compensation. We also found that although most CNAs are trained in injury prevention techniques and rate this training highly, their overall rating of initial training to prepare them for providing direct care in a nursing facility is relatively low.

Examining the relationship between assistive equipment in nursing homes and workplace injuries among CNAs was one of the main goals of this study. Descriptive results show that assistive equipment is widely available and most CNAs use it. However, it appears that equipment use is not necessarily determined by its availability; CNAs are not using the equipment for other reasons. For those few who do not use it or report no access to it, more research is needed to understand why. Importantly, this study found that assistive equipment availability and use do not significantly affect the odds of being injured, once CNA personal and work characteristics are controlled for. This finding is unexpected and is inconsistent with other studies, which found that assistive equipment reduces incidence of injuries, particularly back injuries (Collins et al., 2004; Evanoff, Wolf, Aton, Canos, \& Collins, 2003; Nelson et al., 2006; Silverwood \& Haddock, 2006). On the other hand, studying worker injuries in Ohio nursing homes, Stanev and colleagues (2008) found no statistically significant relationship between worker injury rates and availability of safety equipment.

Lifting equipment by itself may not bring the benefits that are expected from it. It may be too cumbersome to use properly and may require the help of additional staff, who may not be available. It is also possible that, in addition to modern lifting devices, many facilities still have manual crank beds, which require CNAs to bend to position patients. CNAs reported that they often do not have enough time to assist residents with ADLs, so it is also possible that they feel rushed and do not want to wait until the lifts become available or another CNA is available to assist with the lift. Using a lift may be safer, but lifting patients without a lift may be simpler and faster than using assistive equipment.

Several CNA job characteristics are important predictors in this study. New workers-identified as CNAs who are new to direct care work and also new to a facility-are at higher risk of workplace injuries than more experienced CNAs are. Approximately 40 percent of all CNAs nationally may be considered new workers, which is not surprising given the high rate of turnover among direct care staff in nursing facilities (American Health Care Association, 2010). CNA turnover is a major problem for nursing facilities, but is difficult to address.

In addition to new workers, CNAs who reported having two or more jobs in the past 5 years are also at higher risk for all injuries. About three-quarters of all CNAs are in this category nationally. These types of workers may need additional support from the nursing home management and their peers in order to perform their work safely. Decreasing turnover 
may reduce injury rates by retaining CNAs who have learned how to avoid injuries.

Poor job preparation appears to be a serious issue among CNAs. Rating their training as inadequate is common. More than one-third of all CNAs report that they feel unprepared or only somewhat prepared for work in nursing facilities as a result of their initial training (Khatutsky et al., 2007). Our results show that in addition to specific injury prevention techniques, CNAs who report being generally better prepared for the job are able to handle many other challenging aspects of direct care better, resulting in fewer injuries. In addition to dealing with residents with dementia and resident aggression, other job aspects may be relevant to injury, such as ability to follow directions, familiarity with the residents and their care plans, communication skills, discipline, and the ability to balance the need to get tasks done with the need to wait for help from other staff.

This study found that higher wages are associated with decreasing odds of being injured at work. It is possible that wages serve as a proxy for something this model does not directly account for, such as higher motivation and desire to provide better care to nursing home residents. In other work, we have shown that CNA wages are low-about $\$ 10$ per hour on average - and that CNA wage is an important determinant of job tenure (Wiener, Squillace, Anderson, \& Khatutsky, 2009; Khatutsky et al., 2007) and job satisfaction (Bishop, Squillace, Meagher, Anderson, \& Wiener, 2009). However, wage increases for CNAs will most likely require higher reimbursement levels from Medicare and Medicaid, which will be difficult to implement in the current fiscal environment. Stone and Harahan (2010) suggest instituting a payment reform that provides Medicaid rate increases to long-term care employers who reduce turnover, increase retention, and achieve better outcomes through workplace redesign and continuous quality improvement. Achieving an injury-free environment could be one such quality improvement outcome.

CNAs who feel rushed at work and report not having enough time for delivering ADL assistance are also more likely to experience an injury during their work in the facility. More than a third of all CNAs reported not having sufficient time to perform ADL tasks for nursing home residents. It is important to note that this variable measures CNA perception of insufficient time for performing personal care tasks. In contrast, the facility-level CNA staffing ratio was not a significant predictor of injuries, suggesting a more complex relationship between staffing and having time to complete personal care tasks. One of the possibilities is that while staffing ratio determines a caseload for each $\mathrm{CNA}$, it may not account for other factors such as communication and collaborative spirit among CNAs. For example, CNAs may sometimes get injured when they undertake certain tasks alone without waiting for help from their peers, or when they react emotionally to supervisor, resident, or family demands. The ability to balance the need to finish assigned tasks with the need to pace themselves through the work shift and wait for help from other CNAs when it is required may be something that can be taught and emphasized during training.

Overtime work appears to be an important factor in predicting workplace injuries. This finding is consistent with previous research that found overtime, extended work schedules and night shifts increase workers' injuries (Eriksen et al., 2003; Freburger \& Konrad, 2007; Horwitz \& McCall, 2004; Trinkoff, Le, Geiger-Brown, \& Lipscomb, 2007). In all likelihood, CNAs working overtime are tired, which may make them more prone to making mistakes. The need to work overtime may also be related to staffing shortages. If a facility is short on staff, other CNAs may not be available to assist with transferring residents. Overtime work is common in nursing facilities: nationally, about 22 percent of all CNAs report being required to work mandatory overtime, and 88 percent of facilities surveyed by the 2004 NNHS report that their registered nurses, licensed practical nurses, or CNAs worked overtime shifts in the week prior to the survey (Khatutsky et al., 2007).

In this study, we hypothesized that positive and supportive organizational culture in nursing facilities will promote a safer working environment, with fewer injuries. For example, in more collaborative work settings, it may be easier to obtain additional help when needed. Of several measures of CNA perception of organizational culture, two variables were 
significant predictors of injuries: the odds of being injured decreased for CNAs who felt respected and rewarded for their work and for CNAs who felt that the organization values CNA work. Perhaps in these environments, when the management is perceived to be supportive and caring of its employees, workers are more likely to help each other and feel more confident about taking time for various injury prevention measures. Our study is one of the first to show that certain aspects of organizational culture in a facility contribute to a safer work environment by reducing CNA injuries in the workplace.

CNA personal characteristics are included in this study primarily as controls. While not easily changed by policy initiatives or recruitment strategies, certain demographic characteristics create additional vulnerabilities in the work place. Knowing what demographic characteristics appear to increase injuries can help facilities better target their training and interventions. For example, the management may be more insistent that younger and older female CNAs wear special protective back belts when repositioning residents or that they receive more indepth injury prevention training. Finally, contrary to our hypotheses, facility characteristics, such as type of ownership, were not significant predictors of CNA injury.

In terms of study limitations, this study estimates selfreported injury occurrences derived from the NNAS data, which is different from other recent studies that examined occupational injuries reported by nursing facilities to the Occupational Safety and Health Administration (Stanev et al., 2008; Castle et al., 2009). Moreover, the definition of injuries is inclusive: there is no specification that only injuries reported to the nursing facility or requiring medical attention should be listed.

Injuries among CNAs in nursing facilities are very common and may adversely affect job satisfaction, contribute to job shortages, and affect overall quality of care provided by CNAs to nursing facility residents. Injuries are also expensive, as they lead to missed days of work and require shifting CNA staff to lighter duties. This study suggests that several interventions in the area of improving workplace environment may reduce the rates of CNA injury, including the following:

- offering more comprehensive initial training that prepares CNAs for all the aspects of working in nursing facilities, including preventing injury, handling patients safely, dealing with aggressive residents, managing time, and dealing with other issues;

- reducing mandatory overtime;

- providing support, additional training, and mentoring to newly hired CNAs who are also new to the field of direct care; and

- implementing measures to reduce turnover and to better support and educate employees who have a history of high job turnover.

\section{References}

American Health Care Association. (2010). Report of findings 2008 nursing facility staff vacancy, retention, and turnover survey. Washington, DC. Retrieved from http://www.ahcancal.org/ research_data/staffing/Documents/Retention_ Vacancy_Turnover_Survey2008.pdf

Bishop, C. E., Squillace, M. R., Meagher, J., Anderson, W. L., \& Wiener, J. M. (2009). Nursing home work practices and nursing assistants' job satisfaction. The Gerontologist, 49(5), 611-622.
Bureau of Labor Statistics, US Department of Labor. (2007). Occupational injuries and illnesses: Counts, rates, and characteristics, 2006 (Report No. 1014). Retrieved from http://www.bls.gov/iif/oshwc/osh/ os/osh06_cov.pdf

Bureau of Labor Statistics, US Department of Labor. (2011). Workplace injuries and illnesses-2010 (News release USDL-11-1502). October 20, 2011. Retrieved from http://www.bls.gov/news.release/ pdf/osh.pdf 
Castle, N. G., Engberg, J., Mendeloff, J., \& Burns, R. (2009). A national view of workplace injuries in nursing homes. Health Care Management Review, 34(1), 92-103.

Collins, J. W., Wolf, L., Bell, J., \& Evanoff, B. (2004). An evaluation of a "best practices" musculoskeletal injury prevention program in nursing homes. Injury Prevention 10(4), 206-211.

Eriksen, W., Bruusgaard, D., \& Knardahl, S. (2003). Work factors as predictors of sickness absence: a three month prospective study of nurses' aides. Occupational and Environmental Medicine, 60(4), 271-278.

Evanoff, B., Wolf, L., Aton, E., Canos, J., \& Collins, J. (2003). Reduction in injury rates in nursing personnel through introduction of mechanical lifts in the workplace. American Journal of Industrial Medicine, 44(5), 451-457.

Freburger, J. K., \& Konrad, T. R. (2007, April). Musculoskeletal injuries of nursing assistants: The nursing home setting. Paper presented at the 8th Annual UNC's Women's Health Research Day Conference, University of North Carolina at Chapel Hill, NC.

Horwitz, I. B., \& McCall, B. P. (2004). The impact of shift work on the risk and severity of injuries for hospital employees: An analysis using Oregon workers' compensation data. Occupational Medicine, 54(8): 556-563.

Hoskins, A. B. (2006). Occupational injuries, illnesses, and fatalities among nursing, psychiatric, and home health aides, 1995-2004. Compensation and working conditions. Washington, DC: Bureau of Labor Statistics, US Department of Labor. Retrieved from http://www.bls.gov/opub/cwc/ sh20060628ar01p1.htm

Jones, A. L., Dwyer, L. L., Bercovitz, A. R., \& Strahan, G. W. (2009). The National Nursing Home Survey: 2004 overview. National Center for Health Statistics. Vital and Health Statistics, 13(167).
Khatutsky, G., Anderson, W. L., Wiener, J. M., \& Akhmerova, V. (RTI International). (2007, July). Analysis of the National Nursing Assistant Survey (NNAS). Report prepared for the Office of Disability, Aging, and Long-Term Care Policy, Office of the Assistant Secretary for Planning and Evaluation, Department of Health and Human Services, Washington, DC, through Contract Number HHS-100-03-0025, Task Order HHSP23300007T.

Morgan, D. G., Stewart, N. J., D’Arcy, C., Forbes, D., \& Lawson, J. (2005). Work stress and physical assault of nursing aides in rural nursing homes with and without dementia special care units. Journal of Psychiatric and Mental Health Nursing, 12, 347-358.

National Research Council. (2008). Retooling for an aging America: Building the health care workforce. Washington, DC: The National Academies Press.

Nelson, A., Matz, M., Chen, F., Siddharthan, K., Lloyd, J., \& Fragala, G. (2006). Development and evaluation of a multifaceted ergonomics program to prevent injuries associated with patient handling tasks. International Journal of Nursing Studies, 43(6), 717-733.

Silverwood, S., \& Haddock, M. (2006). Reduction of musculoskeletal injuries in intensive care nurses using ceiling-mounted patient lifts. Dynamics, 17(3), 19-21.

Squillace, M. R., Remsburg, R. E., Bercovitz, A., Rosenoff, E., \& Branden, L. (2007). An introduction to the National Nursing Assistant Survey. National Center for Health Statistics. Vital and Health Statistics, 1(44), 1-54.

Stanev, S. S., Bailer A. J., \& Li, H. (2008). Worker injuries in safety equipment in Ohio nursing homes [Brief Report]. Oxford, OH: Scripps Gerontology Center, Miami University.

Stone, R., \& Wiener, J. (2001). Who will care for us? Addressing the LTC workforce crisis. Washington, DC: The Urban Institute and the American Association of Homes and Services for the Aging. 
Stone, R., \& Harahan, M. F. (2010). Improving the long-term care workforce for older adults. Health Affairs, 29(1), 109-115.

Trinkoff, A. M., Le, R., Geiger-Brown, J., \& Lipscomb, J. (2007). Work schedule, needle use, and needlestick injuries among registered nurses. Infection Control and Hospital Epidemiology, 28(2), 156-164.

Trinkoff, A., Johantgen, M., Muntaner, C., \& Le, R. (2005). The relationship between staffing and worker injury in nursing homes. American Journal of Public Health, 95(7), 1220-1225.
Wiener, J., Squillace, M. R., Anderson, W. L., \& Khatutsky, G. (2009). Why do they stay? Job tenure among certified nursing assistants in nursing homes. The Gerontologist, 49(2), 198-210.

Wiener, J. M. (2006). It's not your grandmother's longterm care anymore! Public Policy \& Aging Report, 16(3), 28-35.

Wright, B. (2006). In Brief: Training programs for certified nursing assistants. Washington DC: AARP Public Policy Institute. 


\section{Acknowledgments}

Funding for this paper was provided, in part, by the Retirement Research Foundation, Chicago, Illinois. 
RTI International is an independent, nonprofit research organization dedicated to improving the human condition by turning knowledge into practice. RTI offers innovative research and technical solutions to governments and businesses worldwide in the areas of health and pharmaceuticals, education and training, surveys and statistics, advanced technology, international development, economic and social policy, energy and the environment, and laboratory and chemistry services.

The RTI Press complements traditional publication outlets by providing another way for RTI researchers to disseminate the knowledge they generate. This PDF document is offered as a public service of RTI International. More information about RTI Press can be found at www.rti.org/rtipress. 